\title{
A Posteriori Error Estimates for the Generalized Overlapping Domain Decomposition Methods
}

\author{
Hakima Benlarbi and Ahmed-Salah Chibi \\ Department of Mathematics, Badji Mokhtar University, B.P. 12, Annaba 23000, Algeria \\ Correspondence should be addressed to Ahmed-Salah Chibi, as_chibi@yahoo.fr
}

Received 8 June 2012; Revised 18 September 2012; Accepted 21 November 2012

Academic Editor: Malgorzata Peszynska

Copyright (C) 2012 H. Benlarbi and A.-S. Chibi. This is an open access article distributed under the Creative Commons Attribution License, which permits unrestricted use, distribution, and reproduction in any medium, provided the original work is properly cited.

A posteriori error estimates for the generalized overlapping domain decomposition method (GODDM) (i.e., with Robin boundary conditions on the interfaces), for second order boundary value problems, are derived. We show that the error estimate in the continuous case depends on the differences of the traces of the subdomain solutions on the interfaces. After discretization of the domain by finite elements we use the techniques of the residual a posteriori error analysis to get an a posteriori error estimate for the discrete solutions on subdomains. The results of some numerical experiments are presented to support the theory.

\section{Introduction}

We consider the generalized overlapping domain decomposition method that is, with Robin transmission conditions on the interfaces $[1,2]$ for second order boundary value problems on a bounded domain $\Omega$ with Dirichlet boundary conditions. The a priori estimate of the error is given in several papers, see for instance Lions [3] in which a variational formulation of the classical Schwarz method is derived. In Chan et al. [4] a geometry related convergence results are obtained. Douglas and Huang [1] studied the accelerated version of the GODDM, Engquist and Zhao [2] studied the convergence for simple (rectangular or circular) geometries; however, these authors did not give a criterion to stop the iterative process. All these results can also be found in the recent books on domain decomposition methods of Quarteroni and Valli [5], Toselli and Widlund [6]. Recently Maday and Magoulès $[7,8]$ presented an improved version of the Schwarz method for highly heterogeneous media. This method uses new optimized interface conditions specially designed to take into account the heterogeneity between the subdomains on the interfaces. A recent overview of the current state of the art on domain decomposition methods can be found in two special issues of the 
computer methods in applied mechanics and engineering journal, edited by Farhat and Le Tallec [9], Magoulès and Rixen [10] and in Nataf [11].

In general, the a priori estimate is not suitable for assessing the quality of the approximate solution on subdomains since it depends mainly on the exact solution itself which is unknown. The alternative approach is to use the approximate solution itself in order to find such an estimate. This approach, known as a posteriori estimate, became very popular in the nineties of the last century with finite element methods, see the monographs $[12,13]$ and the references therein. In their paper Otto and Lube [14] gave an a posteriori estimate for a nonoverlapping domain decomposition algorithm that said that "the better the local solutions fit together at the interface the better the errors of the subdomain solutions will be." This error estimate enables us to know with certainty when one must stop the iterative process as soon as the required global precision is reached. A posteriori error analysis was also used by Bernardi et al. [15] to determine an optimal value of the penalty parameter for penalty domain decomposition methods to construct fast solvers.

In various situations it is better to use overlapping decompositions for faster convergence rate, for example, the case of decomposition into simple subdomains where uniform discretizations are possible. So the aim of this paper is to show that a similar result to that in [14], in the case of the GODDM, holds. It is obtained via the introduction of two auxiliary problems defined each over two nonoverlapping subdomains. These auxiliary problems are needed for the analysis and not for the computation.

The paper is organized as follows. In Section 1, we introduce some necessary notations, then we give a variational formulation of our model problem. We establish, in Section 2, a stopping criterion for the iterative process in the continuous case. In Section 3, an a posteriori error estimate is proposed for the convergence of the discretized solution using the finite element method on subdomains. We conclude this section by an adaptation of the techniques of the residual a posteriori error analysis to give an a posteriori estimate in the discrete case.

\subsection{Problem Formulation and Domain Decomposition Method}

Let $\Omega$ be a bounded domain in $\mathbb{R}^{2}$ with a piecewise $C^{1,1}$ boundary $\partial \Omega$. We denote $(\cdot, \cdot)_{\Omega}$ and $\|\cdot\|_{0, \Omega}$ the usual inner product and norm of $L^{2}(\Omega)$. Let $H^{1}(\Omega)$ be the usual Sobolev space with norm $\|\cdot\|_{1, \Omega}$ and seminorm $|\cdot|_{1, \Omega} ; H_{0}^{1}(\Omega)$ is the subspace of functions of $H^{1}(\Omega)$ vanishing on $\partial \Omega$.

Let $\Omega_{1}$ and $\Omega_{2}$ be two subdomains of $\Omega$ (to be defined later), when $\Gamma_{i} \subset \partial \Omega_{i}$ we need the space $W_{i}=H_{00}^{1 / 2}\left(\Gamma_{i}\right)=\left\{\left.v\right|_{\Gamma_{i}}: v \in H^{1}\left(\Omega_{i}\right), v=0\right.$ on $\left.\partial \Omega_{i} \backslash \Gamma_{i}\right\}$ which is a subspace of $H^{1 / 2}\left(\Gamma_{i}\right) ; i=1,2$ equipped with the norm

$$
\|\varphi\|_{W_{i}}=\inf _{v \in H^{1}\left(\Omega_{i}\right), v=\varphi \text { on } \Gamma_{i}}|v|_{1, \Omega_{i}}
$$

On the subdomain $\Omega_{i}$ we use the space $V_{i}=H_{0}^{1}(\Omega) \cap H^{1}\left(\Omega_{i}\right)$ which has as a trace space $W_{i}=\left.V_{i}\right|_{\Gamma_{i}}=H_{00}^{1 / 2}\left(\Gamma_{i}\right), i=1,2$. By $\langle\cdot, \cdot\rangle_{\Gamma_{i}}$ we denote the inner product of $L^{2}\left(\Gamma_{i}\right)$.

Let us consider the following model problem:

$$
\begin{aligned}
-\Delta u & =f \in L^{2}(\Omega), \\
u & =0 \quad \text { on } \partial \Omega,
\end{aligned}
$$


The variational formulation of this problem is given by

$$
\text { Find } u \in V \text { solution of } a(u, v)=(f, v)_{\Omega^{\prime}} \quad \forall v \in V \text {, }
$$

where $a(u, v)=\int_{\Omega} \nabla u \nabla v d x$ and $(f, v)_{\Omega}=\int_{\Omega} f v d x$ for all $v \in V=H_{0}^{1}(\Omega)$. We split the domain $\Omega$ into two overlapping subdomains $\Omega_{1}$ and $\Omega_{2}$ such that

$$
\Omega_{1} \cap \Omega_{2}=\Omega_{12}, \quad \partial \Omega_{i} \cap \Omega_{j}=\Gamma_{i}, \quad i \neq j(i, j=1,2) .
$$

The generalized overlapping domain decomposition method is written as follows: set $u_{i}^{0}$ in $\Omega_{i}$ and construct the sequence $u_{i}^{n+1}, i=1,2$ in parallel for $n=0,1,2, \ldots$

$$
\begin{aligned}
& -\Delta u_{1}^{n+1}=f \quad \text { in } \Omega_{1}, \\
& u_{1}^{n+1}=0 \quad \text { on } \partial \Omega_{1} \cap \partial \Omega, \\
& \frac{\partial u_{1}^{n+1}}{\partial \eta_{1}}+\alpha_{1} u_{1}^{n+1}=\frac{\partial u_{2}^{n}}{\partial \eta_{1}}+\alpha_{1} u_{2}^{n} \quad \text { on } \Gamma_{1}, \\
& -\Delta u_{2}^{n+1}=f \quad \text { in } \Omega_{2}, \\
& u_{2}^{n+1}=0 \quad \text { on } \partial \Omega_{2} \cap \partial \Omega, \\
& \frac{\partial u_{2}^{n+1}}{\partial \eta_{2}}+\alpha_{2} u_{2}^{n+1}=\frac{\partial u_{1}^{n}}{\partial \eta_{2}}+\alpha_{2} u_{1}^{n} \quad \text { on } \Gamma_{2},
\end{aligned}
$$

where $\eta_{i}$ is the exterior normal to $\Omega_{i}$ and $\alpha_{i}$ is a real parameter, $i=1,2$.

The convergence of the GODDM (1.5) and (1.6) is proved in $[2,5]$. Our main interest is to obtain an a posteriori error estimate we need for stopping the iterative process as soon as the required global precision is reached.

The weak formulations of problems (1.5), (1.6) are given, respectively, by

$$
\begin{aligned}
& u_{1}^{n+1} \in V_{1}: a_{1}\left(u_{1}^{n+1}, v_{1}\right)+\left\langle\alpha_{1} u_{1}^{n+1}, v_{1}\right\rangle_{\Gamma_{1}}=\left(f, v_{1}\right)+\left\langle\frac{\partial u_{2}^{n}}{\partial \eta_{1}}+\alpha_{1} u_{2}^{n}, v_{1}\right\rangle_{\Gamma_{1}}, \quad \forall v_{1} \in V_{1}, \\
& u_{2}^{n+1} \in V_{2}: a_{2}\left(u_{2}^{n+1}, v_{1}\right)+\left\langle\alpha_{2} u_{2}^{n+1}, v_{1}\right\rangle_{\Gamma_{2}}=\left(f, v_{2}\right)+\left\langle\frac{\partial u_{1}^{n}}{\partial \eta_{2}}+\alpha_{2} u_{1}^{n}, v_{1}\right\rangle_{\Gamma_{2}}, \quad \forall v_{2} \in V_{2},
\end{aligned}
$$

where $V_{i}$ is defined above. It can also be written as

$$
V_{i}=\left\{v \in H^{1}\left(\Omega_{i}\right):\left.v\right|_{\partial \Omega_{i} \cap \partial \Omega}=0\right\}, \quad a_{i}(u, v)=(\nabla u, \nabla v)_{\Omega_{i}} .
$$

\section{A Posteriori Error Estimate in the Continuous Case}

Since it is numerically easier to compare the subdomain solutions on the interfaces $\Gamma_{1}$ and $\Gamma_{2}$ rather than on the overlap $\Omega_{12}$, thus we need to introduce two auxiliary problems defined on 


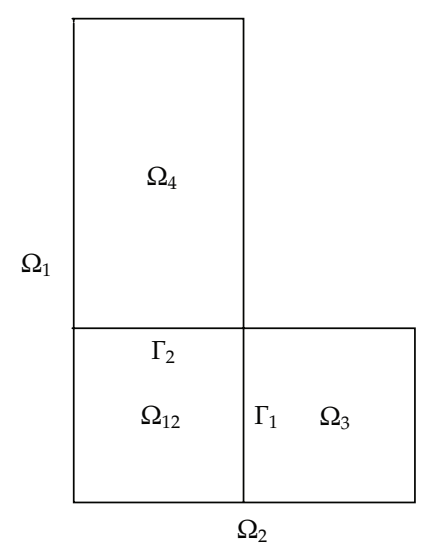

(a)

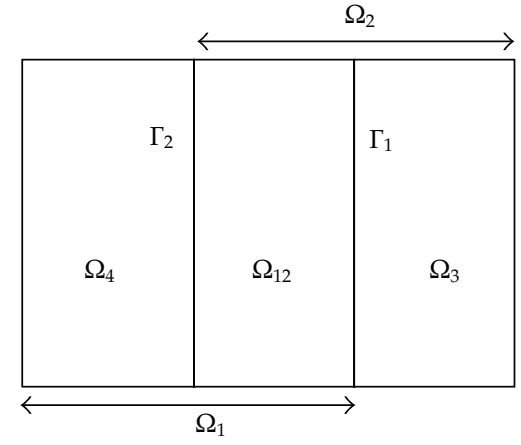

(b)

Figure 1: Two examples of domain decompositions (Schur and Schwarz).

nonoverlapping subdomains of $\Omega$. This idea allows us to obtain the a posteriori error estimate by following the steps of Otto and Lube [14]. We get these auxiliary problems by coupling each one of the problems (1.5) and (1.6) with another problem in a nonoverlapping way over $\Omega$. These auxiliary problems are needed for the analysis and not for the computation, to get the estimate. We see that we can go from the GODDM to a couple of nonoverlapping domain decomposition methods with Robin boundary conditions on the interfaces $\Gamma_{1}$ and $\Gamma_{2}$. To define these auxiliary problems we need to split the domain $\Omega$ into two sets of disjoint subdomains: $\left(\Omega_{1}, \Omega_{3}\right)$ and $\left(\Omega_{2}, \Omega_{4}\right)$, see Figure 1 , such that

$$
\Omega=\Omega_{1} \cup \Omega_{3}, \quad \text { with } \Omega_{1} \cap \Omega_{3}=\emptyset, \quad \Omega=\Omega_{2} \cup \Omega_{4} \quad \text { with } \Omega_{2} \cap \Omega_{4}=\emptyset \text {. }
$$

Let $\left(u_{1}^{n+1}, u_{2}^{n+1}\right)$ be the solutions of problems (1.5), (1.6). We define the couple $\left(u_{1}^{n+1}, u_{3}^{n+1}\right)$ over $\left(\Omega_{1}, \Omega_{3}\right)$ to be the solution of the following nonoverlapping problems:

$$
\begin{aligned}
& -\Delta u_{1}^{n+1}=f \quad \text { in } \Omega_{1}, \\
& u_{1}^{n+1}=0 \quad \text { on } \partial \Omega_{1} \cap \partial \Omega, \\
& \frac{\partial u_{1}^{n+1}}{\partial \eta_{1}}+\alpha_{1} u_{1}^{n+1}=\frac{\partial u_{2}^{n}}{\partial \eta_{1}}+\alpha_{1} u_{2}^{n} \quad \text { on } \Gamma_{1}, \\
& -\Delta u_{3}^{n+1}=f \quad \text { in } \Omega_{3}, \\
& u_{3}^{n+1}=0 \quad \text { in } \partial \Omega_{3} \cap \partial \Omega, \\
& \frac{\partial u_{3}^{n+1}}{\partial \eta_{3}}+\alpha_{3} u_{3}^{n+1}=\frac{\partial u_{1}^{n}}{\partial \eta_{3}}+\alpha_{3} u_{1}^{n} \text { on } \Gamma_{1} .
\end{aligned}
$$

We can write $u_{2}^{n}=u_{3}^{n}+\epsilon_{1}^{n}$ on $\Gamma_{1}$ (in the 3rd equation in (2.2)) that is, $\epsilon_{1}^{n}$ is the difference between the overlapping and the nonoverlapping solutions $u_{2}^{n}$ and $u_{3}^{n}$ (in problems (1.5)-(1.6) 
and (2.2)-(2.3) resp.,) in $\Omega_{3}$. Because both overlapping and nonoverlapping problems converge, see $[2,5]$ that is, $u_{2}^{n}$ and $u_{3}^{n}$ tend to $u_{2}=\left.u\right|_{\Omega_{2}}, \epsilon_{1}^{n}$ should tend to naught an $n$ tends to infinity in $V_{2}$.

By multiplying the first equation in (2.2) by $v_{1} \in V_{1}$ and integrating by parts we obtain

$$
\int_{\Omega_{1}} \nabla u_{1}^{n+1} \nabla v_{1} d x+\int_{\Gamma_{1}} \alpha_{1} u_{1}^{n+1} v_{1} d x=\int_{\Omega_{1}} f v_{1} d x+\int_{\Gamma_{1}} \Lambda_{3}^{n} v_{1} d \sigma
$$

where $\Lambda_{3}^{n}$ is given by

$$
\Lambda_{3}^{n}=\frac{\partial u_{3}^{n}}{\partial \eta_{1}}+\alpha_{1} u_{3}^{n}+\frac{\partial \epsilon_{1}^{n}}{\partial \eta_{1}}+\alpha_{1} \epsilon_{1}^{n}
$$

On the other hand, using the last equation in (2.3) and putting

$$
\theta_{1}^{n}=\frac{\partial \epsilon_{1}^{n}}{\partial \eta_{1}}+\alpha_{1} \epsilon_{1}^{n}
$$

we get

$$
\begin{aligned}
\Lambda_{3}^{n+1} & =\frac{\partial u_{3}^{n+1}}{\partial \eta_{1}}+\alpha_{1} u_{3}^{n+1}+\theta_{1}^{n+1}=-\frac{\partial u_{3}^{n+1}}{\partial \eta_{3}}+\alpha_{1} u_{3}^{n+1}+\theta_{1}^{n+1} \\
& =\alpha_{3} u_{3}^{n+1}-\frac{\partial u_{1}^{n}}{\partial \eta_{3}}-\alpha_{3} u_{1}^{n}+\alpha_{1} u_{3}^{n+1}+\theta_{1}^{n+1} \\
& =\left(\alpha_{1}+\alpha_{3}\right) u_{3}^{n+1}-\Lambda_{1}^{n}+\zeta^{n}
\end{aligned}
$$

where $\zeta^{n}=\theta_{1}^{n+1}+\theta_{3}^{n}$. From this result we can write the following algorithm which is equivalent to the auxiliary nonoverlapping problem (2.2)-(2.3). We need this algorithm and two lemmas for obtaining an a posteriori error estimate for this problem.

Algorithm 2.1. The sequences $\left(u_{1}^{n+1}, u_{3}^{n+1}\right)_{n \in \mathbb{N}}$ solutions of problems (2.2), (2.3) satisfy the following domain decomposition algorithm denoted ALG.G.D.D.M.

(1) Let $\Lambda_{i}^{0} \in W_{1}^{*}$ be an initial value, $i=1,3$ ( $W_{1}^{*}$ is the dual of $W_{1}$ ).

(2) Given $\Lambda_{j}^{n} \in W_{1}^{*}$ solve for $i=1,3, i \neq j$ : Find $u_{i}^{n+1} \in V_{i}$ solutions of

$$
a_{i}\left(u_{i}^{n+1}, v_{i}\right)+\left\langle\alpha_{i} u_{i}^{n+1}, v_{i}\right\rangle_{\Gamma_{1}}=F\left(v_{i}\right)+\left\langle\Lambda_{j}^{n}, v_{i}\right\rangle_{\Gamma_{1}}, \quad \forall v_{i} \in V_{i}
$$

(3) Compute new data $\Lambda_{i}^{n+1} \in W_{1}^{*}, i=1,3$ from

$$
\left\langle\Lambda_{i}^{n+1}, \varphi\right\rangle_{\Gamma_{1}}=\left\langle\left(\alpha_{i}+\alpha_{j}\right) u_{i}^{n+1}, \varphi\right\rangle_{\Gamma_{1}}-\left\langle\Lambda_{j}^{n}, \varphi\right\rangle_{\Gamma_{1}}+\left\langle\zeta^{n}, \varphi\right\rangle_{\Gamma_{1}} \quad \forall \varphi \in W_{1}, j \neq i .
$$

(4) Set $n=n+1$, go to Step 2 . 
Lemma 2.2. Let $u_{i}=\left.u\right|_{\Omega_{i}}, e_{i}^{n+1}=u_{i}^{n+1}-u_{i}, \eta_{i}^{n}=\Lambda_{i}^{n}-\Lambda_{i}$ and $\xi_{1}^{n}=\zeta^{n}-\zeta$. Then for $i=1,3, i \neq j$, the following relations hold

$$
\begin{gathered}
a_{i}\left(e_{i}^{n+1}, v_{i}\right)+\left\langle\alpha_{i} e_{i}^{n+1}, v_{i}\right\rangle_{\Gamma_{1}}=\left\langle\eta_{j}^{n}, v_{i}\right\rangle_{\Gamma_{1}}, \quad \forall v_{i} \in V_{i}, \\
\left\langle\eta_{i}^{n+1}, \varphi\right\rangle_{\Gamma_{1}}=\left\langle\left(\alpha_{i}+\alpha_{j}\right) e_{i}^{n+1}, \varphi\right\rangle_{\Gamma_{1}}-\left\langle\eta_{j}^{n}, \varphi\right\rangle_{\Gamma_{1}}+\left\langle\xi_{1}^{n}, \varphi\right\rangle_{\Gamma_{1}}, \quad \forall \varphi \in W_{1} .
\end{gathered}
$$

The proof follows directly from steps 2 and 3 of ALG.G.D.D.M.

Lemma 2.3. By letting $C$ be a generic constant which has different values at different places one get for $i=1,3, i \neq j$

$$
\begin{aligned}
& \left\langle\eta_{j}^{n-1}-\alpha_{i} e_{i}^{n}, w\right\rangle_{\Gamma_{1}} \leq C\left\|e_{i}^{n}\right\|_{1, \Omega_{i}}\|w\|_{W_{1}} \\
& \left\langle\alpha_{i} w+\xi_{1}^{n}, e_{i}^{n+1}\right\rangle_{\Gamma_{1}} \leq C\left\|e_{i}^{n+1}\right\|_{1, \Omega_{i}}\|w\|_{W_{1}} .
\end{aligned}
$$

Proof. Using (2.10) and the fact that the trace mapping $\operatorname{Tr}_{i}: V_{i} \rightarrow W_{i}$ and its inverse are continuous, we obtain

$$
\begin{aligned}
\left\langle\eta_{j}^{n-1}-\alpha_{i} e_{i}^{n}, w\right\rangle_{\Gamma_{1}} & =a_{i}\left(e_{i}^{n}, \operatorname{Tr}_{i}^{-1} w\right)=\left(\nabla e_{i}^{n}, \nabla \operatorname{Tr}_{i}^{-1} w\right) \\
& \leq\left|e_{i}^{n}\right|_{1, \Omega_{i}}\left|\operatorname{Tr}_{i}^{-1} w\right|_{1, \Omega_{i}} \\
& \leq C_{\operatorname{Tr}_{i}^{-1}}\left\|e_{i}^{n}\right\|_{1, \Omega_{i}}\|w\|_{W_{1}} .
\end{aligned}
$$

On the otherhand we have

$$
\begin{aligned}
\left\langle\alpha_{i} w+\xi_{1}^{n} e_{i}^{n+1}\right\rangle_{\Gamma_{1}} & =\int_{\Gamma_{1}}\left(\alpha_{i} w+\xi_{1}^{n}\right) e_{i}^{n+1} d s \\
& \leq\left[\left|\alpha_{i}\right|\|w\|_{0, \Gamma_{1}}+\left|\xi_{1}^{n}\right|_{0, \Gamma_{1}}\right]\left\|e_{i}^{n+1}\right\|_{0, \Gamma_{1}} \\
& \leq \max \left(\left|\alpha_{i}\right|,\left|\xi_{1}^{n}\right|_{0, \Gamma_{1}}\right)\|w\|_{0, \Gamma_{1}}\left\|e_{i}^{n+1}\right\|_{0, \Gamma_{1}} \\
& \leq C\left\|e_{i}^{n+1}\right\|_{1, \Omega_{i}}\|w\|_{W_{1}} .
\end{aligned}
$$

This ends the proof.

Based on the previous two lemmas, we can obtain the following a posteriori error estimate for the nonoverlapping domain decomposition problem (2.2)-(2.3). 
Proposition 2.4. For the sequences $\left(u_{1}^{n+1}, u_{3}^{n+1}\right)_{n \in \mathbb{N}}$ solutions of problems (2.2), (2.3) one has the following a posteriori error estimation

$$
\left\|e_{1}^{n+1}\right\|_{1, \Omega_{1}}+\left\|e_{3}^{n}\right\|_{1, \Omega_{3}} \leq C\left\|u_{1}^{n+1}-u_{3}^{n}\right\|_{H_{00}^{1 / 2}\left(\Gamma_{1}\right)} .
$$

Proof. From (2.10) and (2.11) we have

$$
\begin{aligned}
a_{1}\left(e_{1}^{n+1}, v_{1}\right)+a_{3}\left(e_{3}^{n}, v_{3}\right) & =\left\langle\eta_{3}^{n}-\alpha_{1} e_{1}^{n+1}, v_{1}\right\rangle_{\Gamma_{1}}+\left\langle\eta_{1}^{n-1}-\alpha_{3} e_{3}^{n}, v_{3}\right\rangle_{\Gamma_{1}} \\
& =\left\langle\alpha_{1}\left(e_{3}^{n}-e_{1}^{n+1}\right)+\xi_{1}^{n}, v_{1}\right\rangle_{\Gamma_{1}}+\left\langle\eta_{1}^{n-1}-\alpha_{3} e_{3}^{n}, v_{3}-v_{1}\right\rangle_{\Gamma_{1}} .
\end{aligned}
$$

Taking $v_{1}=e_{1}^{n+1}$ and $v_{2}=e_{2}^{n}$, then using Lemma 2.3 we get

$$
\begin{aligned}
\frac{1}{2}\left(\left\|e_{1}^{n+1}\right\|_{1, \Omega_{1}}+\left\|e_{3}^{n}\right\|_{1, \Omega_{3}}\right)^{2} & \leq\left\|e_{1}^{n+1}\right\|_{1, \Omega_{1}}^{2}+\left\|e_{3}^{n}\right\|_{1, \Omega_{3}}^{2}=a_{1}\left(e_{1}^{n+1}, e_{1}^{n+1}\right)+a_{3}\left(e_{3}^{n}, e_{3}^{n}\right) \\
& \leq\left\langle\alpha_{1}\left(e_{3}^{n}-e_{1}^{n+1}\right)+\xi_{1}^{n}, e_{1}^{n+1}\right\rangle_{\Gamma_{1}}+\left\langle\eta_{1}^{n-1}-\alpha_{3} e_{3}^{n}, e_{3}^{n}-e_{1}^{n+1}\right\rangle_{\Gamma_{1}} \\
& \leq C_{1}\left\|e_{1}^{n+1}\right\|_{1, \Omega_{1}}\left\|e_{3}^{n}-e_{1}^{n+1}\right\|_{H_{00}^{1 / 2}\left(\Gamma_{1}\right)}+C_{2}\left\|e_{3}^{n}\right\|_{1, \Omega_{3}}\left\|e_{3}^{n}-e_{1}^{n+1}\right\|_{H_{00}^{1 / 2}\left(\Gamma_{1}\right)} \\
& \leq C\left\{\left\|e_{1}^{n+1}\right\|_{1, \Omega_{1}}+\left\|e_{3}^{n}\right\|_{1, \Omega_{3}}\right\}\left\|u_{1}^{n+1}-u_{3}^{n}\right\|_{H_{00}^{1 / 2}\left(\Gamma_{1}\right)^{\prime}}
\end{aligned}
$$

which is the required result.

In the similar way, we define another nonoverlapping auxiliary problem over $\left(\Omega_{2}, \Omega_{4}\right)$. The sequences $\left(u_{2}^{n+1}, u_{4}^{n+1}\right)_{n \in \mathbb{N}}$ are solutions of

$$
\begin{aligned}
& -\Delta u_{2}^{n+1}=f \quad \text { in } \Omega_{2}, \\
& u_{2}^{n+1}=0 \quad \text { on } \partial \Omega_{2} \cap \partial \Omega, \\
& \frac{\partial u_{2}^{n+1}}{\partial \eta_{2}}+\alpha_{2} u_{2}^{n+1}=\frac{\partial u_{1}^{n}}{\partial \eta_{2}}+\alpha_{2} u_{1}^{n} \quad \text { on } \Gamma_{2}, \\
& -\Delta u_{4}^{n+1}=f \quad \text { in } \Omega_{4}, \\
& u_{4}^{n+1}=0 \quad \text { on } \partial \Omega_{4} \cap \partial \Omega, \\
& \frac{\partial u_{4}^{n+1}}{\partial \eta_{4}}+\alpha_{4} u_{4}^{n+1}=\frac{\partial u_{2}^{n}}{\partial \eta_{4}}+\alpha_{4} u_{2}^{n} \quad \text { on } \Gamma_{2},
\end{aligned}
$$

where we can write $u_{1}^{n}=u_{4}^{n}+\epsilon_{2}^{n}$ on $\Gamma_{2}$ (in the 3rd equation in (2.18)) that is, $\epsilon_{2}^{n}$ is the difference between the overlapping and the nonoverlapping solutions $u_{1}^{n}$ and $u_{4}^{n}$ (in problems (1.5)-(1.6) 
and (2.18)-(2.19) resp.) in $\Omega_{4}$. As both sequences $u_{1}^{n}$ and $u_{4}^{n}$ converge towards $u_{1}, \epsilon_{2}^{n}$ should tend to naught as $n$ tends to infinity. Using Lemmas 2.2 and 2.3 over $\left(\Omega_{2}, \Omega_{4}\right)$ we obtain the $a$ posteriori error estimate for this auxiliary problem.

Proposition 2.5. For the sequences $\left(u_{2}^{n+1}, u_{4}^{n+1}\right)_{n \in \mathbb{N}}$ solutions of problems (2.18), (2.19) one has the following a posteriori error estimate

$$
\left\|u_{2}^{n+1}-u_{2}\right\|_{1, \Omega_{2}}+\left\|u_{4}^{n}-u_{4}\right\|_{1, \Omega_{4}} \leq C\left\|u_{2}^{n+1}-u_{4}^{n}\right\|_{H_{00}^{1 / 2}\left(\Gamma_{2}\right)} .
$$

Remark 2.6. We remark from Propositions 2.4 and 2.5 that we can go from sequences of solutions to problems (1.5) and (1.6) to sequences of solutions to problems generated by nonoverlapping domain decomposition problems of Robin type transmission conditions across the interfaces $\Gamma_{1}$ and $\Gamma_{2}$. This result enable us to compare the subdomain iterations of the GODDM on the interfaces $\Gamma_{1}$ and $\Gamma_{2}$ rather than on the overlap region $\Omega_{12}$.

In the following theorem we are going to use this fact to obtain an a posteriori error estimate for the continuous generalized overlapping domain decomposition method (1.5)(1.6).

Theorem 2.7. Let $u_{i}=u \Omega_{\Omega_{i}}$ where $u$ is the solution of problem (1.2). For the sequences $\left(u_{1}^{n+1}, u_{2}^{n+1}\right)_{n \in \mathbb{N}}$ solutions of problems (1.5) and (1.6), one has the following a posteriori error estimate

$$
\left\|u_{1}^{n+1}-u_{1}\right\|_{1, \Omega_{1}}+\left\|u_{2}^{n}-u_{2}\right\|_{1, \Omega_{2}} \leq C\left\{\begin{array}{c}
\left\|u_{1}^{n+1}-u_{2}^{n}\right\|_{H_{00}^{1 / 2}\left(\Gamma_{1}\right)}+\left\|u_{2}^{n}-u_{1}^{n-1}\right\|_{H_{00}^{1 / 2}\left(\Gamma_{2}\right)} \\
+\left\|\epsilon_{1}^{n}\right\|_{H_{00}^{1 / 2}\left(\Gamma_{1}\right)}+\left\|\epsilon_{2}^{n-1}\right\|_{H_{00}^{1 / 2}\left(\Gamma_{2}\right)}
\end{array}\right\} .
$$

Proof. We use two auxiliary problems defined each over two nonoverlapping subdomains of $\Omega$. These two problems are defined over $\left(\Omega_{1}, \Omega_{3}\right)$ and $\left(\Omega_{2}, \Omega_{4}\right)$, respectively. From the previous two propositions we have

$$
\begin{aligned}
\left\|u_{1}^{n+1}-u_{1}\right\|_{1, \Omega_{1}}+\left\|u_{2}^{n}-u_{2}\right\|_{2, \Omega_{2}} \leq & \left\|u_{1}^{n+1}-u_{1}\right\|_{1, \Omega_{1}}+\left\|u_{3}^{n}-u_{3}\right\|_{1, \Omega_{3}} \\
& +\left\|u_{2}^{n}-u_{2}\right\|_{1, \Omega_{2}}+\left\|u_{4}^{n-1}-u_{4}\right\|_{1, \Omega_{4}} \\
\leq & C\left\|u_{1}^{n+1}-u_{3}^{n}\right\|_{H_{00}^{1 / 2}\left(\Gamma_{1}\right)}+C\left\|u_{2}^{n}-u_{4}^{n-1}\right\|_{H_{00}^{1 / 2}\left(\Gamma_{2}\right)} .
\end{aligned}
$$

From the definitions of $\epsilon_{1}^{n}$ and $\epsilon_{2}^{n}$, we have

$$
\begin{aligned}
\left\|u_{1}^{n+1}-u_{1}\right\|_{1, \Omega_{1}}+\left\|u_{2}^{n}-u_{2}\right\|_{1, \Omega_{2}} \leq & C\left\|u_{2}^{n}-u_{1}^{n-1}-\epsilon_{2}^{n-1}\right\|_{H_{00}^{1 / 2}\left(\Gamma_{2}\right)}+C\left\|u_{1}^{n+1}-u_{2}^{n}-\epsilon_{1}^{n}\right\|_{H_{00}^{1 / 2}\left(\Gamma_{1}\right)} \\
\leq C & \left\{\left\|u_{1}^{n+1}-u_{2}^{n}\right\|_{H_{00}^{1 / 2}\left(\Gamma_{1}\right)}+\left\|u_{2}^{n}-u_{1}^{n-1}\right\|_{H_{00}^{1 / 2}\left(\Gamma_{2}\right)}\right. \\
+ & \left.\left\|\epsilon_{1}^{n}\right\|_{H_{00}^{1 / 2}\left(\Gamma_{1}\right)}+\left\|\epsilon_{2}^{n-1}\right\|_{H_{00}^{1 / 2}\left(\Gamma_{2}\right)}\right\},
\end{aligned}
$$

which is the required result. 


\section{A Posteriori Error Estimate in the Discrete Case}

In this section, we consider the discretization of the variational problems (1.7) and (1.8). Let $\tau_{h}$ be a triangulation of $\Omega$ compatible with the decomposition and $V_{h} \subset V$ is the subspace of continuous functions (polynomials of degree $k, k \geq 1$ ) which vanish over $\partial \Omega$. We have

$$
V_{i, h}=\left.V_{h}\right|_{\Omega_{i}}, \quad W_{i, h}=\left.V_{h}\right|_{\Gamma_{i}}
$$

$W_{i, h}$ is a subspace of $H_{00}^{1 / 2}\left(\Gamma_{i}\right)$ which consists of continuous piecewise polynomial functions on $\Gamma_{i}$ which vanish at the end points of $\Gamma_{i}(i=1,2)$.

Let $u_{h} \in V_{h}$ be the solution of the discrete problem associated with (1.3), $u_{i, h}=\left.u_{h}\right|_{\Omega_{i}}$. We construct the sequences $\left(u_{i, h}^{n+1}\right)_{i=1,2}, u_{i, h}^{n+1} \in V_{i, h}$ solutions of the discrete versions of (1.5) and (1.6) at the $(n+1)$ th iteration, that is,

$$
\begin{aligned}
& -\Delta u_{1, h}^{n+1}=f \quad \text { on } \Omega_{1}, \\
& u_{1, h}^{n+1}=0 \quad \text { on } \partial \Omega_{1} \cap \partial \Omega, \\
& \frac{\partial u_{1, h}^{n+1}}{\partial \eta_{1}}+\alpha_{1} u_{1, h}^{n+1}=\frac{\partial u_{2, h}^{n}}{\partial \eta_{1}}+\alpha_{1} u_{2, h}^{n} \quad \text { on } \Gamma_{1}, \\
& -\Delta u_{2, h}^{n+1}=f \quad \text { on } \Omega_{2}, \\
& u_{2, h}^{n+1}=0 \quad \text { on } \partial \Omega_{2} \cap \partial \Omega, \\
& \frac{\partial u_{2, h}^{n+1}}{\partial \eta_{2}}+\alpha_{2} u_{2, h}^{n+1}=\frac{\partial u_{1, h}^{n}}{\partial \eta_{2}}+\alpha_{2} u_{1, h}^{n} \quad \text { on } \Gamma_{2} .
\end{aligned}
$$

In a similar manner to that of Section 2, we introduce two discrete auxiliary problems, one over $\left(\Omega_{1}, \Omega_{3}\right)$, the other over $\left(\Omega_{2}, \Omega_{4}\right)$. We let

$$
\begin{aligned}
& -\Delta u_{1, h}^{n+1}=f \quad \text { in } \Omega_{1}, \\
& u_{1, h}^{n+1}=0 \quad \text { on } \partial \Omega_{1} \cap \partial \Omega, \\
& \frac{\partial u_{1, h}^{n+1}}{\partial \eta_{1}}+\alpha_{1} u_{1, h}^{n+1}=\frac{\partial u_{2, h}^{n}}{\partial \eta_{1}}+\alpha_{1} u_{2, h}^{n} \quad \text { on } \Gamma_{1}, \\
& -\Delta u_{3, h}^{n+1}=f \quad \text { in } \Omega_{3}, \\
& u_{3, h}^{n+1}=0 \quad \text { on } \partial \Omega_{3} \cap \partial \Omega, \\
& \frac{\partial u_{3, h}^{n+1}}{\partial \eta_{3}}+\alpha_{3} u_{1, h}^{n+1}=\frac{\partial u_{1, h}^{n}}{\partial \eta_{3}}+\alpha_{3} u_{1, h}^{n} \quad \text { on } \Gamma_{1}
\end{aligned}
$$

We can write $u_{2, h}^{n}=u_{3, h}^{n}+\epsilon_{1, h}^{n}$ on $\Gamma_{1}$, (in the 3rd equation in (3.4)), that is, $\epsilon_{1, h}^{n}$ is the difference between the discrete overlapping and the nonoverlapping solutions $u_{2, h}^{n}$ and $u_{3, h}^{n}$ (in problems 
(3.2)-(3.3) and (3.4)-(3.5), resp.) in $\Omega_{3}$. Because both $u_{2, h}^{n}$ and $u_{3, h}^{n}$ converges to $u_{2,} \epsilon_{1, h}^{n}$ should tend to naught as $n$ tends to infinity.

Similarly, over $\left(\Omega_{2}, \Omega_{4}\right)$ we consider the sequences $\left(u_{2, h}^{n+1}, u_{4, h}^{n+1}\right)_{n \in \mathbb{N}}$ solutions of the following discrete problems:

$$
\begin{aligned}
& -\Delta u_{2, h}^{n+1}=f \quad \text { in } \Omega_{2,} \\
& u_{2, h}^{n+1}=0 \quad \text { on } \partial \Omega_{2} \cap \partial \Omega, \\
& \frac{\partial u_{2, h}^{n+1}}{\partial \eta_{2}}+\alpha_{2} u_{2, h}^{n+1}=\frac{\partial u_{1, h}^{n}}{\partial \eta_{2}}+\alpha_{2} u_{1, h}^{n} \quad \text { on } \Gamma_{2,} \\
& -\Delta u_{4, h}^{n+1}=f \quad \text { in } \Omega_{4}, \\
& u_{4, h}^{n+1}=0 \quad \text { on } \partial \Omega_{4} \cap \partial \Omega, \\
& \frac{\partial u_{4, h}^{n+1}}{\partial \eta_{4}}+\alpha_{4} u_{4, h}^{n+1}=\frac{\partial u_{2, h}^{n}}{\partial \eta_{4}}+\alpha_{4} u_{2, h}^{n} \quad \text { on } \Gamma_{2},
\end{aligned}
$$

where we can also write $u_{1, h}^{n}=u_{4, h}^{n}+\epsilon_{2, h}^{n}$ on $\Gamma_{2,}, \epsilon_{2, h}^{n}$ tends to naught as $n$ tends to infinity as explained for the previous problems. We can obtain the discrete counterparts of Propositions 2.4 and 2.5 by doing almost the same analysis as in Section 2 (i.e., passing from continuous spaces to discrete subspaces and from continuous sequences to discrete ones). Therefore

$$
\begin{aligned}
& \left\|u_{1, h}^{n+1}-u_{1, h}\right\|_{1, \Omega_{1}}+\left\|u_{3, h}^{n}-u_{3, h}\right\|_{1, \Omega_{3}} \leq C\left\|u_{1, h}^{n+1}-u_{3, h}^{n}\right\|_{W_{1, h}}, \\
& \left\|u_{2, h}^{n+1}-u_{2, h}\right\|_{1, \Omega_{2}}+\left\|u_{4, h}^{n}-u_{4, h}\right\|_{1, \Omega_{4}} \leq C\left\|u_{2, h}^{n+1}-u_{4, h}^{n}\right\|_{W_{2, h}{ }^{\prime}}
\end{aligned}
$$

where the discrete trace subspace norm $\|\cdot\|_{W_{i, h}}$ defined over $\Gamma_{i}(i=1,2)$ is taken to be

$$
\left\|w_{h}\right\|_{W_{i, h}}^{2}=\int_{\Gamma_{i}} w_{h}^{2} d x+\iint_{\Gamma_{i}} \frac{\left(w_{h}(x)-w_{h}(y)\right)^{2}}{|x-y|^{2}} d x d y
$$

Hence, using the previous two inequalities, we have

$$
\left\|u_{1, h}^{n+1}-u_{1, h}\right\|_{1, \Omega_{1}}+\left\|u_{2, h}^{n}-u_{2, h}\right\|_{1, \Omega_{2}} \leqslant C\left\{\begin{array}{c}
\left\|u_{2, h}^{n}-u_{1, h}^{n-1}\right\|_{W_{2, h}}+\left\|u_{1, h}^{n+1}-u_{2, h}^{n}\right\|_{W_{1, h}} \\
+\left\|\epsilon_{1, h}^{n}\right\|_{W_{1, h}}+\left\|\epsilon_{2, h}^{n-1}\right\|_{W_{2, h}}
\end{array}\right\}
$$

We remark that (3.10) is the discrete version of (2.21).

Remark 3.1. Let us note that (3.10) is an estimate of the error between the approximate solution $u_{i, h}$ and the approximate solution of the discretized overlapping domain decomposition algorithm $u_{i, h}^{n+1}, i=1,2$. 
Next we will obtain an estimate of the error between the approximate solution $u_{i, h}^{n+1}$ and the exact solution $u$. We introduce some necessary notations. We denote by

$$
\varepsilon_{h}=\left\{E \text { a side of } \tau_{h} ; E \notin \partial \Omega\right\} .
$$

For every $T \in \tau_{h}$ and $E \in \varepsilon_{h}$, we define

$$
\omega_{T}=\cup\left\{T^{\prime} \in \tau_{h} ; T^{\prime} \cap T \neq \phi\right\}, \quad \omega_{E}=\cup\left\{T^{\prime} \in \tau_{h} ; T^{\prime} \cap E \neq \phi\right\} .
$$

For a function $f$ which is not necessarily continuous across two neighboring elements of $\tau_{h}$ having $E$ as a common side, $[f]$ denotes the jump of $f$ across $E$ and $\eta_{E}$ the normal vector of E.

We have the following theorem which gives the a posteriori error estimate for the discrete GODDM.

Theorem 3.2. Let $u_{i}=\left.u\right|_{\Omega_{i}}$ where $u$ is the solution of problem (1.2), the sequences $\left(u_{1, h}^{n+1}, u_{2, h}^{n+1}\right)_{n \in \mathbb{N}}$ are solutions of problems (3.2)-(3.3). Then there exists a constant $C$ independent of $h$ such that

$$
\left\|u_{1, h}^{n+1}-u_{1}\right\|_{1, \Omega_{1}}+\left\|u_{2, h}^{n}-u_{2}\right\|_{1, \Omega_{2}} \leq C\left\{\sum_{i=1}^{2}\left(\sum_{T \in \tau_{h}}\left(\eta_{i}^{T}\right)^{2}+h_{T}^{2}\left\|f-f_{h}\right\|_{0, T}^{2}\right)^{1 / 2}+\sum_{i=1}^{2} \eta_{\Gamma_{i}}\right\},
$$

where

$$
\begin{aligned}
& \eta_{\Gamma_{i}}=\left\|u_{i, h}^{*}-u_{j, h}^{*-1}\right\|_{W_{i, h}}+\left\|\epsilon_{i, h}^{*}\right\|_{W_{i, h}{ }^{\prime}} \quad i, j=1,2, i \neq j, \\
& \eta_{i}^{T}=h_{T}\left\|f_{h}+\Delta u_{i, h}^{*}\right\|_{0, T}+\sum_{E \in \varepsilon_{h}} h_{E}^{1 / 2}\left\|\left[\frac{\partial u_{i, h}^{*}}{\partial \eta_{E}}\right]\right\|_{0, E} \cdot
\end{aligned}
$$

The symbol $*$ corresponds to $n+1$ when $i=1$ and to $n$ when $i=2$.

Proof. The proof is based on the technique of the residual a posteriori estimation (see [13]) and Theorem 2.7. We give the main steps. By the triangle inequality we have

$$
\sum_{i=1}^{2}\left\|u_{i}-u_{i, h}^{*}\right\|_{1, \Omega_{i}} \leq \sum_{i=1}^{2}\left\|u_{i}-u_{i, h}\right\|_{1, \Omega_{i}}+\sum_{i=1}^{2}\left\|u_{i, h}-u_{i, h}^{*}\right\|_{1, \Omega_{i}} .
$$

The second term on the right-hand side of (3.15) is bounded as in (3.10) by

$$
\sum_{i=1}^{2}\left\|u_{i, h}-u_{i, h}^{*}\right\|_{1, \Omega_{i}} \leq C \sum_{i=1}^{2}\left(\left\|u_{i}^{*}-u_{j}^{*-1}\right\|_{W_{i, h}}+\left\|\epsilon_{i, h}^{*}\right\|_{W_{i, h}}\right) .
$$


To bound the first term on the r.h.s of (3.15) we use the residual equation and apply the technique of the residual a posteriori error estimation, see [13], to get for $v_{h} \in V_{h}$ and $v_{i, h}=$ $\left.v_{h}\right|_{\Omega_{i}}$

$$
\begin{aligned}
a_{i}\left(u_{i}-u_{i, h}, v\right)= & a_{i}\left(u_{i}-u_{i, h}, v-v_{h}\right) \\
= & \sum_{T \subset \Omega_{i}} \int\left(f_{h}+\Delta u_{i, h}\right)\left(v_{i}-v_{i, h}\right)-\sum_{E \subset \Omega_{i}} \int_{E}\left[\frac{\partial u_{i, h}}{\partial \eta_{E}}\right]\left(v_{i}-v_{i, h}\right) d s \\
& -\sum_{E \subset \Gamma_{i}} \int_{E} \frac{\partial u_{i, h}}{\partial \eta_{E}}\left(v_{i}-v_{i, h}\right) d s+\sum_{T \subset \Omega_{i}}\left(f-f_{h}, v_{i}-v_{i, h}\right)_{\Omega_{i}}+\left\langle\frac{\partial u_{i}}{\partial \eta_{i}}, u-v_{h}\right\rangle_{\Gamma_{i}},
\end{aligned}
$$

where $f_{h}$ is any approximation of $f$ by polynomials of degree at most $k$, therefore

$$
\begin{gathered}
\sum_{i=1}^{2} a_{i}\left(u_{i}-u_{i, h}, v\right) \leq \sum_{i=1}^{2}\left\{\begin{array}{c}
\sum_{T \subset \Omega_{i}}\left\|f+\Delta u_{i, h}\right\|_{0, T}\left\|v_{i}-v_{i, h}\right\|_{0, T} \\
+\sum_{E \subset \Omega_{i}}\left\|\left[\frac{\partial u_{i, h}}{\partial \eta_{E}}\right]\right\|_{0, E}\left\|v_{i}-v_{i, h}\right\|_{0, E} \\
+\sum_{E \subset \Gamma_{i}}\left\|\frac{\partial u_{i, h}}{\partial \eta_{E}}\right\|_{0, E}\left\|v_{i}-v_{i, h}\right\|_{0, E} \\
+\sum_{T \subset \Omega_{i}}\left\|f-f_{h}\right\|_{0, T}\left\|v_{i}-v_{i, h}\right\|_{0, T} \\
+\left\|\frac{\partial u_{i}}{\partial \eta_{i}}\right\|\left\|_{0, \Gamma_{i}}\right\| u-v_{h} \|_{0, \Gamma_{i}}
\end{array}\right\} \\
\leq \sum_{i=1}^{2}\left(\sum_{T \subset \Omega_{i}}\left(\eta_{i}^{T}\right)^{2}+h_{T}^{2}\left\|f-f_{h}\right\|_{0, T}^{2}\right)^{1 / 2} \sum_{i=1}^{2}\left|v_{i}\right|_{1, \Omega_{i}} .
\end{gathered}
$$

We use the fact that

$$
\left\|u_{i}-u_{i, h}\right\|_{1, \Omega_{i}} \leq \sup _{v \in V_{i}} \frac{a_{i}\left(u_{i}-u_{i, h}, v\right)}{\|v\|_{1, \Omega_{i}}}
$$

Finally, by combining (3.15), (3.18), and (3.19) the required result follows.

\subsection{Numerical Examples}

As an illustration of the theoretical results obtained in this article and to be able to use the $a$ posteriori error estimate (3.10), we consider the following problem

$$
\begin{aligned}
& -\Delta u=f \in L^{2}(\Omega), \\
& u=0 \quad \text { on } \partial \Omega,
\end{aligned}
$$

with $\Omega=] 0,1\left[^{2}\right.$ for the first and second examples and $\left.\Omega=\right] 0,1[\times] 0,1 / 2[\cup] 0,1 / 2[\times] 0,1[$ for the third example (i.e., an $L$-shaped region). The exact solution is taken to be 
Table 1: Results on the square without $a$ posteriori estimates as a stopping criteria for the first example.

\begin{tabular}{lcccc}
\hline$h$ & $1 / 8$ & $1 / 16$ & $1 / 32$ & $1 / 64$ \\
\hline Er $_{1}$ & $1.7957693(-02)$ & $8.1821001(-03)$ & $4.5210766(-03)$ & $3.1683034(-03)$ \\
Er $_{2}$ & $1.0956427(-02)$ & $9.6854433(-03)$ & $8.7326244(-03)$ & $6.9200024(-03)$ \\
Trer $_{1}$ & $2.2513498(-02)$ & $0.1504199(-02)$ & $8.1897348(-03)$ & $3.4817880(-02)$ \\
Trer $_{2}$ & $1.7198801(-02)$ & $2.1677755(-02)$ & $9.3967836(-03)$ & $6.9538120(-03)$ \\
Iterations & 12 & 10 & 7 & 5 \\
Quotient & 0.7280898 & 0.7507522 & 0.7536285 & 0.9667203 \\
\hline
\end{tabular}

$u(x, y)=x y(x-1)(y-1) e^{x y}$ for the first example, $u(x, y)=\sin 8 \pi x \cdot \sin 8 \pi y$ for the second example, and finally $u(x, y)=x y(x-1)(y-1)(x-1 / 2)(y-1 / 2)$ for the third example.

We first compute the bilinear Galerkin solution $u_{h}$ over $\Omega$ and then apply the generalized overlapping domain decomposition method (1.7)-(1.8) to compute the bilinear sequences $u_{i, h}^{n+1}(i=1,2)$ to be able to look at the behavior of the constant $C$ within (3.10) as the mesh size becomes small $\left(h^{-1}=8,16,32,64\right)$. We take $\left.\Omega_{1}=\right] 0,3 / 4[\times] 0,1[$ and $\left.\Omega_{2}=\right] 1 / 4,1[\times] 0,1[$ for the first and second examples. As for the third example we have $\left.\Omega_{1}=\right] 0,1[\times] 0,1 / 2\left[\right.$ and $\left.\Omega_{2}=\right] 0,1 / 2[\times] 0,1[$. We denote by

$$
\begin{aligned}
& \operatorname{Er}_{i}=\left\|u_{i, h}-u_{i, h}^{n}\right\|_{1, \Omega_{i}{ }^{\prime}} \\
& \operatorname{Trer}_{1}=\left\|u_{1, h}^{n+1}-u_{2, h}^{n}\right\|_{W_{1, h}{ }^{\prime}} \\
& \operatorname{Trer}_{2}=\left\|u_{2, h}^{n}-u_{1, h}^{n-1}\right\|_{W_{2, h}{ }^{\prime}} \\
& \text { Quotient }=\frac{\left(\operatorname{Er}_{1}+\mathrm{Er}_{2}\right)}{\left(\operatorname{Trer}_{1}+\operatorname{Trer}_{2}\right)} .
\end{aligned}
$$

The generalized overlapping domain decomposition method, with $\alpha_{1}=\alpha_{2}=0.5$, converges. The iterations have been stopped when the relative error between two subsequent iterates is less than $\epsilon=10^{-6}$.

The Quotient in Tables 1, 2, 3, 4, 5, and 6 represents an estimate of the constant $C$ in (3.10). It happens to tend to a constant value around 1 which what we expect (i.e., we have asymptotic exactness). We present, for each example, results with and without the a posteriori estimate as a stopping criteria. We see that we obtain the same results but with less iterations when using the a posteriori estimate as a stopping criteria for a moderately small mesh size for the first and third examples. But for the second example, the difference in iteration count is clear between the two approaches.

\subsection{Concluding Remarks}

(1) From Theorem 3.2 we see that the subdomain errors depend on the differences of the latter on the interface, on the data $f$, and on the approximate solution $u^{h}$. The first two terms of (3.13) are the contribution to the a posteriori error from the finite element discretization whereas the last term is the domain decomposition 
Table 2: Results on the square with a posteriori estimates as a stopping criteria for the first example.

\begin{tabular}{lcccc}
\hline$h$ & $1 / 8$ & $1 / 16$ & $1 / 32$ & $1 / 64$ \\
\hline $\mathrm{Er}_{1}$ & $1.3457623(-02)$ & $4.1221001(-03)$ & $8.5210766(-03)$ & $3.1683034(-03)$ \\
$\mathrm{Er}_{2}$ & $1.0556422(-02)$ & $5.2454431(-03)$ & $8.4322244(-03)$ & $6.2200024(-03)$ \\
Trer $_{1}$ & $2.2513498(-02)$ & $5.1504199(-03)$ & $2.1897348(-02)$ & $3.4817880(-03)$ \\
Trer $_{2}$ & $2.4423401(-02)$ & $6.1677755(-03)$ & $1.3967836(-03)$ & $6.9538120(-03)$ \\
Iterations & 3 & 5 & 6 & 7 \\
Quotient & 0.5116240 & 0.7276534 & 0.7277927 & 0.8996421 \\
\hline
\end{tabular}

Table 3: Results on the square without a posteriori estimates as a stopping criteria for the second example.

\begin{tabular}{lcccc}
\hline$h$ & $1 / 8$ & $1 / 16$ & $1 / 32$ & $1 / 64$ \\
\hline Er $_{1}$ & $1.7957693(-02)$ & $2.5598288(-03)$ & $4.7627655(-03)$ & $0.8936322(-04)$ \\
Er $_{2}$ & $1.0956427(-02)$ & $4.5353208(-02)$ & $8.1970235(-03)$ & $6.3020810(-03)$ \\
Trer $_{1}$ & $2.2513498(-02)$ & $5.2993342(-02)$ & $1.1897348(-02)$ & $3.4817880(-03)$ \\
Trer $_{2}$ & $1.7198801(-08)$ & $6.0068501(-09)$ & $1.3967836(-08)$ & $6.9538120(-08)$ \\
Iterations & 10 & 9 & 7 & 5 \\
Quotient & 0.7280898 & 0.9041330 & 1.089299 & 1.560316 \\
\hline
\end{tabular}

Table 4: Results on the square with a posteriori estimates as a stopping criteria for the second example.

\begin{tabular}{lcccc}
\hline$h$ & $1 / 8$ & $1 / 16$ & $1 / 32$ & $1 / 64$ \\
\hline Er $_{1}$ & $4.7345582(-02)$ & $8.1821001(-04)$ & $4.8510591(-03)$ & $1.4817880(-02)$ \\
Er $_{2}$ & $1.3897220(-02)$ & $9.6854433(-02)$ & $8.1924358(-03)$ & $6.9200024(-03)$ \\
Trer $_{1}$ & $9.6471474(-02)$ & $0.1504199(-02)$ & $1.1897348(-02)$ & $1.4817880(-02)$ \\
Trer $_{2}$ & $9.1859622(-09)$ & $0.0000000(+00)$ & $0.3967836(-09)$ & $2.9538120(-08)$ \\
Iterations & 8 & 6 & 5 & 4 \\
Quotient & 0.6348280 & 0.6493334 & 1.096336 & 1.018248 \\
\hline
\end{tabular}

Table 5: Results on the $L$-shaped region without a posteriori estimates as a stopping criteria.

\begin{tabular}{lcccc}
\hline$h$ & $1 / 8$ & $1 / 16$ & $1 / 32$ & $1 / 64$ \\
\hline $\mathrm{Er}_{1}$ & $3.5915386(-02)$ & $16.3642002(-03)$ & $9.0421132(-03)$ & $6.3366068(-03)$ \\
$\mathrm{Er}_{2}$ & $2.19128497(-02)$ & $19.3708866(-03)$ & $12.4732488(-03)$ & $9.8440048(-03)$ \\
Trer $_{1}$ & $4.50217996(-02)$ & $0.2008398(-02)$ & $4.3794696(-02)$ & $2.9635760(-02)$ \\
Trer $_{2}$ & $3.4397602(-02)$ & $4.3355510(-02)$ & $2.7935672(-03)$ & $5.9076240(-03)$ \\
Iterations & 10 & 8 & 6 & 6 \\
Quotient & 0.5443332 & 0.6562642 & 0.8380865 & 1.008344 \\
\hline
\end{tabular}

Table 6: Results on the $L$-shaped region with a posteriori estimates as a stopping criteria.

\begin{tabular}{lcccc}
\hline$h$ & $1 / 8$ & $1 / 16$ & $1 / 32$ & $1 / 64$ \\
\hline Er $_{1}$ & $2.5815386(-02)$ & $14.3642002(-03)$ & $9.0421132(-03)$ & $4.3366122(-03)$ \\
Er $_{2}$ & $2.19128487(-02)$ & $12.3708866(-03)$ & $12.4732488(-03)$ & $6.8440223(-03)$ \\
Trer $_{1}$ & $4.60217446(-02)$ & $0.4008398(-02)$ & $4.3794696(-02)$ & $4.8635660(-03)$ \\
Trer $_{2}$ & $3.3397402(-02)$ & $2.3355510(-02)$ & $2.7935672(-03)$ & $6.8076220(-03)$ \\
Iterations & 4 & 5 & 6 & 7 \\
Quotient & 0.6009663 & 0.9170200 & 0.8380865 & 0.9579689 \\
\hline
\end{tabular}


error. Numerical results indicate that the finite element part of the error estimate dominates the domain decomposition part.

(2) As far as the asymptotic exactness of the estimator based on (3.13) is concerned, Ainsworth and Oden in [12, Theorem 3.5] gave a necessary condition for this property to hold. This condition says that the local residuals in (3.13) should be computed exactly.

(3) The a posteriori error analysis for a second order boundary value problem or Stokes problem is possible. Also, similar results can be obtained in the general case of more than two subdomains.

\section{References}

[1] J. Douglas, Jr. and C.-S. Huang, "An accelerated domain decomposition procedure based on Robin transmission conditions," BIT Numerical Mathematics, vol. 37, no. 3, pp. 678-686, 1997.

[2] B. Engquist and H.-K. Zhao, "Absorbing boundary conditions for domain decomposition," Applied Numerical Mathematics, vol. 27, no. 4, pp. 341-365, 1998.

[3] P.-L. Lions, "On the Schwarz alternating method. I," in First International Symposium on Domain Decomposition Methods for Partial Differential Equations, R. Glowinski, G. H. Golub, G. A. Meurant, and J. Périaux, Eds., pp. 1-42, SIAM, Philadelphia, Pa, USA, 1988.

[4] T. F. Chan, T. Y. Hou, and P.-L. Lions, "Geometry related convergence results for domain decomposition algorithms," SIAM Journal on Numerical Analysis, vol. 28, no. 2, pp. 378-391, 1991.

[5] A. Quarteroni and A. Valli, Domain Decomposition Methods for Partial Differential Equations, The Clarendon Press, Oxford, UK, 1999.

[6] A. Toselli and O. Widlund, Domain Decomposition Methods-Algorithms and Theory, vol. 34 of Springer Series in Computational Mathematics, Springer, Berlin, Germany, 2005.

[7] Y. Maday and F. Magoulès, "Improved ad hoc interface conditions for Schwarz solution procedure tuned to highly heterogeneous media," Applied Mathematical Modelling, vol. 30, no. 8, pp. 731-743, 2006.

[8] Y. Maday and F. Magoulès, "A survey of various absorbing interface conditions for the Schwarz algorithm tuned to highly heterogeneous media," in Domain Decomposition Methods: Theory and Applications, vol. 25 of Gakuto International Series. Mathematical Sciences Applications, pp. 65-93, Gakkōtosho, Tokyo, Japan, 2006.

[9] C. Farhat and P. Le Tallec, "Vista in Domain Decomposition Methods," Computer Methods in Applied Mechanics and Engineering, vol. 184, no. 2-4, pp. 143-520, 2000.

[10] F. Magoulès and D. Rixen, "Domain decomposition methods: recent advances and new challenges in engineering," Computer Methods in Applied Mechanics and Engineering, vol. 196, no. 8, pp. 1345-1346, 2007.

[11] F. Nataf, "Recent developments on optimized Schwarz methods," in Domain Decomposition Methods in Science and Engineering XVI, vol. 55 of Lecture Notes in Computational Science and Engineering, pp. 115-125, Springer, Berlin, Germany, 2007.

[12] M. Ainsworth and J. T. Oden, A Posteriori Error Estimation in Finite Element Analysis, WileyInterscience, New York, NY, USA, 2000.

[13] A. Verfürth, A Review of a Posteriori Error Estimation and Adaptive Mesh-Refinement Techniques, Wiley Teubner, Stuttgart, Germany, 1996.

[14] F.-C. Otto and G. Lube, "A posteriori estimates for a non-overlapping domain decomposition method," Computing, vol. 62, no. 1, pp. 27-43, 1999.

[15] C. Bernardi, T. Chacón Rebollo, E. Chacón Vera, and D. Franco Coronil, "A posteriori error analysis for two-overlapping domain decomposition techniques," Applied Numerical Mathematics, vol. 59, no. 6, pp. 1214-1236, 2009. 


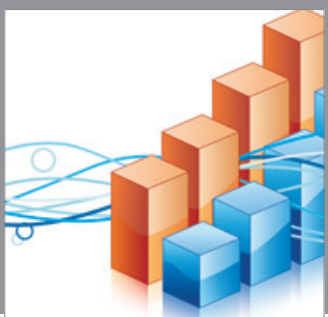

Advances in

Operations Research

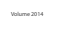

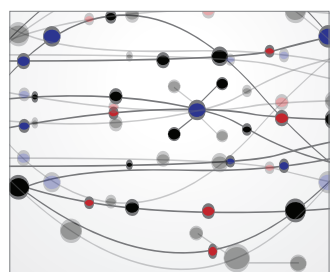

\section{The Scientific} World Journal
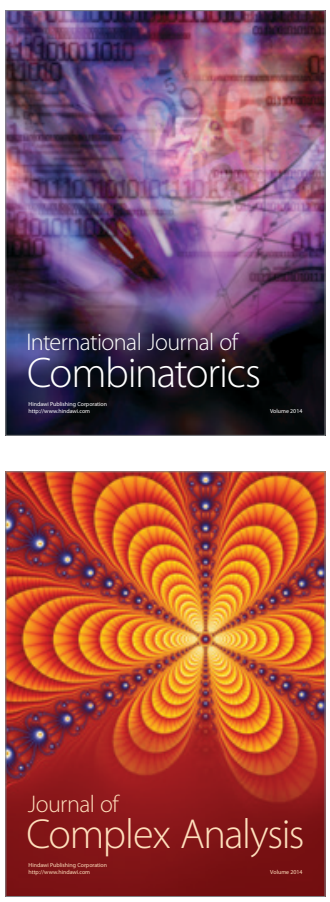

International Journal of

Mathematics and

Mathematical

Sciences
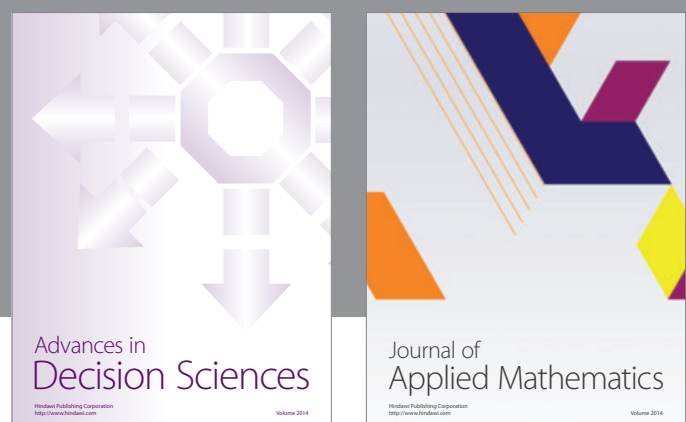

Journal of

Applied Mathematics
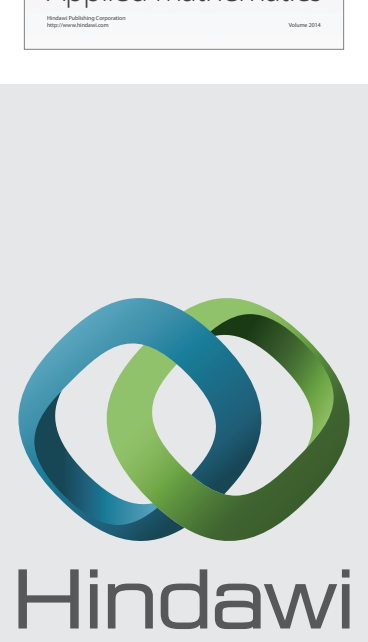

Submit your manuscripts at http://www.hindawi.com
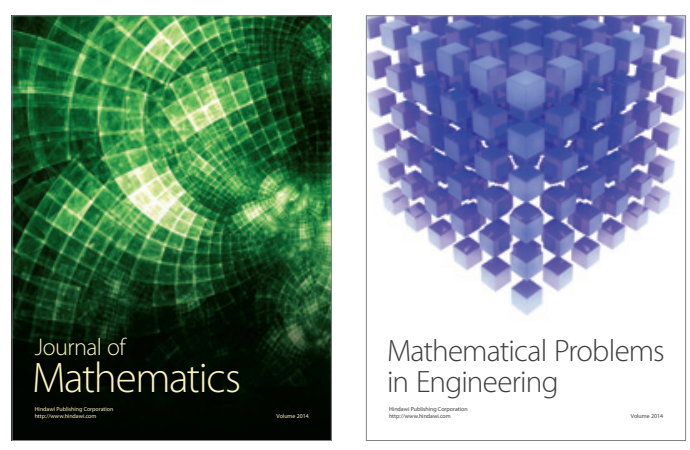

Mathematical Problems in Engineering
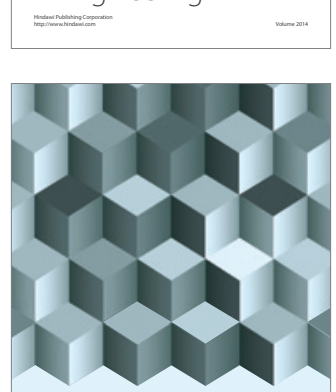

Journal of

Function Spaces
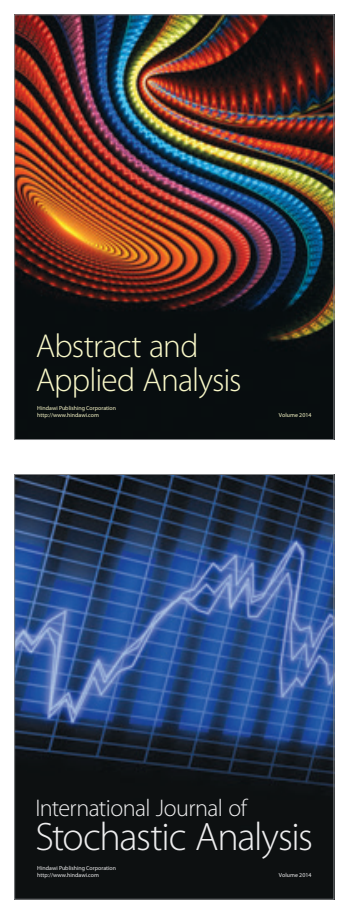

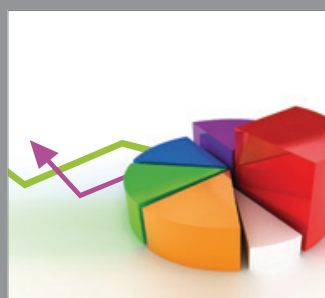

ournal of

Probability and Statistics

Promensencen
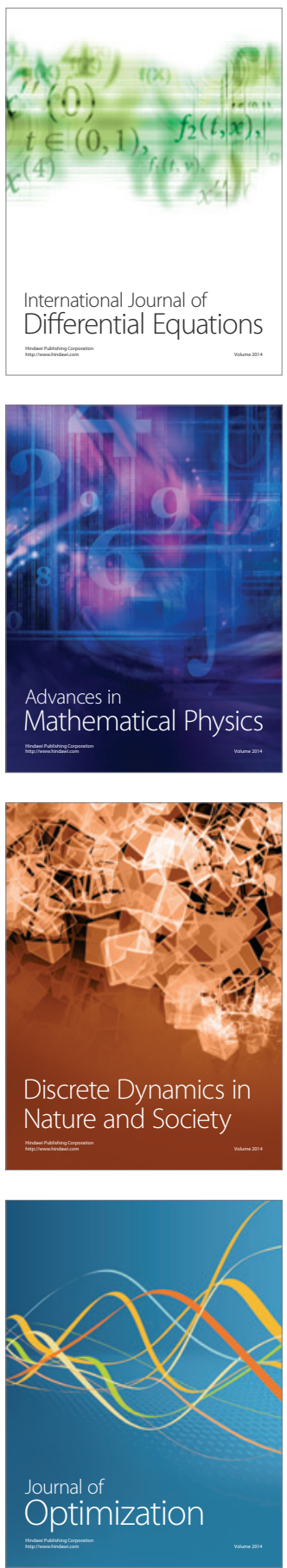